\title{
THREE-LAYER HELE-SHAW DISPLACEMENT WITH AN INTERMEDIATE NON-NEWTONIAN FLUID
}

\author{
Gelu Paşa \\ Simion Stoilow Institute of Mathematics \\ of Romanian Academy \\ Calea Grivitei 21, Bucharest S1, ROMANIA
}

\begin{abstract}
We study the displacement of two Stokes immiscible fluids in a porous medium, approximated by the Hele-Shaw horizontal model. An intermediate non-Newtonian polymer-solute, whose viscosity is depending on the velocity, is considered between the initial fluids. The linear stability problem of this three-layer displacement does not make sense. If the intermediate viscosity depends on velocity and on the polymer concentration, we can obtain a minimization of the Saffman-Taylor instability.
\end{abstract}

AMS Subject Classification: 34B09; 34D20; 35C09; 35J20; 76S05; 76A05 Key Words: Hele-Shaw displacements; non-Newtonian fluids; hydrodynamic stability

\section{Introduction}

We consider a displacing fluid (water) with constant viscosity $\mu_{W}>0$, which is pushing a second immiscible fluid (oil) with constant viscosity $\mu_{O}>\mu_{W}$, in a horizontal Hele-Shaw cell. A well-known result is given by Saffman and Taylor [15]: the flow is unstable.

An intermediate non-Newtonian liquid, with a variable viscosity depending on the velocity, is considered between water and oil. We study the effect of the intermediate liquid on the flow stability.

Received: February 2, 2021

(c) 2021 Academic Publications 
The Hele-Shaw cell is parallel with the fix plane $x_{1} O y$. The flow is from the left to the right, due to the water velocity $U$ far upstream $\left(x_{1} \rightarrow-\infty\right)$. In the moving coordinate system $x=x_{1}-U t$, the displacing and displaced fluids are contained in the regions $x<-L$ and $x>0$. The intermediate region is the interval $-L<x<0$ and contains the non-Newtonian liquid. The $O z$ axis is orthogonal on the plates. The gravity effects are neglected.

As usual in the Hele-Shaw model, between the above three immisicble fluids we have two sharp interfaces. We prove that the system which governs the linear stability of the interfaces of this three-layer displacement does not make sense.

Some previous results were obtained when the middle region is filled by a polymer solution with variable viscosity, which is function of the polymer concentration $c$, or is verifying a diffusion equation. In the first case - see [3], $[6],[7],[10]$ - it was proved that an "optimal" viscosity profile exists, which can give a significant improvement of the stability. In the second case - see [4], [5], [11] - it was proved that the diffusion is improving the stability, compared with the Saffman-Taylor case, when the viscosity profile is verifying some specific conditions.

In [14] we proved that several intermediate Stokes liquids with constant viscosities, inserted between the initial fluids, can not minimize the SaffmanTaylor instability.

The use of polymer-solute in the middle region is related with liquids which are non-Newtonian. The relation between tangential stress and deformation tensor is not linear, or/and the viscosity in the middle region depends on some parameters of the flow.

When an Oldroyd-B fluid is displaced by air or two Olrdroyd-B fluids are displacing in a Hele-Shaw cell, the interfaces are much more unstable compared with the Saffman-Taylor displacement - see [12] and [16].

In Section 2 we consider an intermediate polymer-solute whose viscosity is depending only on the displacing velocity $U$ far upstream. The linear stability problem does not make sense.

In Section 3 we consider an intermediate viscosity depending on $U$ and on the polymer concentration $c$. With some additional conditions, we get a stabilizing effect, compared with the Saffman-Taylor case.

\section{When $\mu$ is depending on $U$}

A non-Newtonian model of a liquid with viscosity depending on the velocity was proposed in [8]. A Darcy's law type was obtained for a shear-rate dependent 
viscosity. This model can be used to justify the flows studied in [1], [2].

Consider a horizontal Hele-Shaw cell with the gap $b$ between the plates. The pressure, the averaged velocities (across the gap $b$ ) and the viscosity are denoted by $p,(u, v, w), \mu$. The component $w$ is neglected. The following equations are considered (in the cited above papers) for the flow in the middle region:

$$
\begin{gathered}
\nabla_{x, y} p=-\mu\left(\mathbf{u}^{2} / b^{2}\right) \mathbf{u}, \quad p_{z}=0, \\
\mathbf{u}=(u, v), \quad u_{x}+v_{y}=0, \\
\mu\left(\mathbf{u}^{2}\right)=\mu_{S} \frac{1+a \mathbf{u}^{2} \tau}{1+\mathbf{u}^{2} \tau} \cdot \frac{b^{2}}{12}, \\
d \mu / d \theta=\mu_{S} \frac{\tau(a-1)}{(1+\theta \tau)^{2}} \cdot \frac{b^{2}}{12}, \quad \theta=\mathbf{u}^{2},
\end{gathered}
$$

where $\tau$ is a characteristic time. The parameter $a$ is governing the sign of $d \mu / d \theta$. We have shear thinning for $a<1$ and shear thickening for $a>1$.

In [8] is given the following explanation for the Darcy type law (1). A dependence of $\mu$ on the square of the trace of the rate-of-strain-tensor is assumed. For a Poiseuille flow, that means $\mu$ is function of $\mathbf{u}_{\mathbf{z}}{ }^{2}$.

The first step is to use the general form of the flow equations - see for example [9]. Thus the unsteady flow is governed by the equations

$$
\begin{gathered}
\mathbf{u}_{t}=-\nabla p+\left[\mu(\phi) \mathbf{u}_{z}\right]_{z}=-\nabla p+\eta \mathbf{u}_{z z}, \\
\eta(\phi)=[\mu(\phi)+2 \phi d \mu / d \phi], \quad \phi=\mathbf{u}_{z}^{2},
\end{gathered}
$$

where $\eta$ is the "effective viscosity". We need a positive $\eta$. To this end we impose the condition

$$
\mu(\phi)+2 \phi d \mu / d \phi>0 .
$$

Therefore, in the steady $2 \mathrm{D}$ case, the general flow equations with viscosity depending on the rate-of-strain tensor are

$$
\nabla_{2} p=\left\{\mu\left(\mathbf{u}_{\mathbf{z}}^{\mathbf{2}}\right) \mathbf{u}_{\mathbf{z}}\right\}_{z}, \quad \mathbf{u}=(u, v), \quad u_{x}+v_{y}=0,
$$

where the index 2 on the $\nabla$ operator stands for $x, y$ derivatives. We integrate with respect to $z$ and get (recall $p$ is not depending on $z$ )

$$
z \nabla_{2} p=\mu\left(\mathbf{u}_{\mathbf{z}}^{\mathbf{2}}\right) \mathbf{u}_{\mathbf{z}} .
$$

The second step is to find $\mathbf{u}_{z}$ as function of $\nabla_{2} p$. The invertibility of the above relation near $\mathbf{u}_{z}=0$, by using the implicit function theorem, is possible only if

$$
h_{\psi}(0)>0, \quad h(\psi)=\psi \mu\left(\psi^{2}\right), \quad \psi=\mathbf{u}_{\mathbf{z}} .
$$


We have $h_{\psi}=\mu\left(\psi^{2}\right)+2 \psi^{2} \mu_{\psi}\left(\psi^{2}\right)$, therefore the invertibility condition is exactly the inequality (4). The inversion procedure is performed in [8]. The last step for obtaining the Darcy type law (1) is the average procedure of the velocity across the Hele-Shaw gap. Thus $\mathbf{u}$ is obtained in terms of the pressure gradient.

We consider the basic solution with two straight interfaces:

$$
\begin{gathered}
x_{L}=-L, \quad x_{R}=0 ; \\
u=U, v=0 ; \quad P_{x}=-\mu U ; P_{y}=0 ; \\
\mu=\mu_{W}, x<-L ; \quad \mu=\mu_{O}, x>0 ; \\
\mu_{W}<\mu=\mu\left(U^{2}\right)<\mu_{O}, \quad x \in(-L, 0) .
\end{gathered}
$$

In this section we study the linear stability of the solution (7).

The small perturbations are denoted by $u^{\prime}, v^{\prime}, p^{\prime}, \mu^{\prime}$.

The viscosity perturbation $\mu^{\prime}$ is obtained as follows. In the frame of the linear stability analysis, we neglect $u^{\prime 2}, v^{\prime 2}$ and get

$$
E:=\mu\left(\left(U+u^{\prime}\right)^{2}+v^{\prime 2}\right) \approx \mu\left(U^{2}+2 U u^{\prime}\right) .
$$

The first-order Taylor expansion is giving

$$
\begin{aligned}
& E=\mu\left(U^{2}\right)+2 U u^{\prime} \cdot \mu_{\theta}\left(U^{2}\right):=\mu(\theta)+\mu^{\prime}, \\
& \mu^{\prime}:=2 U \mu_{\theta} u^{\prime}, \quad \theta=U^{2}, \quad \mu_{\theta}=d \mu / d \theta .
\end{aligned}
$$

We insert $u^{\prime}, v^{\prime}, p^{\prime}, \mu^{\prime}$ in the flow equations (1) and obtain

$$
\begin{gathered}
\left(P+p^{\prime}\right)_{x}=-\left(\mu+\mu^{\prime}\right)\left(U+u^{\prime}\right), \\
\left(P+p^{\prime}\right)_{y}=-\left(\mu+\mu^{\prime}\right)\left(v^{\prime}\right), \\
\left(u+u^{\prime}\right)_{x}+\left(v+v^{\prime}\right)_{y}=0 \Rightarrow \\
u_{x}^{\prime}+v_{y}^{\prime}=0, \\
p_{x}^{\prime}=-\mu(\theta) u^{\prime}-2 \theta \mu_{\theta} u^{\prime}, \\
p_{y}^{\prime}=-\mu(\theta) v^{\prime} .
\end{gathered}
$$

We use a Fourier decomposition for the velocity perturbation

$$
u^{\prime}(x, y, t)=f(x) \exp (i k y+\sigma t)
$$


and from (10) and (12) we get

$$
\begin{gathered}
v^{\prime}=-\left[f_{x} / i k\right] \exp (i k y+\sigma t), \\
p^{\prime}=-\mu(\theta)\left[f_{x} / k^{2}\right] \exp (i k y+\sigma t) .
\end{gathered}
$$

By cross derivation of the pressure perturbations (11) and (12) it follows the amplitude equation:

$$
\begin{aligned}
& {\left[\mu(\theta) u^{\prime}+2 \theta \mu_{\theta} u^{\prime}\right]_{y}=\left[\mu(\theta) v^{\prime}\right]_{x}} \\
& \Rightarrow-\mu f_{x x}+k^{2}\left(\mu+2 \theta \mu_{\theta}\right) f=0 .
\end{aligned}
$$

The above equation is quite similar with the relation (11) of [14], but the coefficient of $f$ is $k^{2}\left(\mu+2 \theta \mu_{\theta}\right)$ instead of $k^{2}$.

The relation (14) holds for all $x \in R x \neq-L, x \neq 0$.

We use the relation (2) and get

$$
\begin{gathered}
\mu+2 \theta \mu_{\theta}=\left[\frac{1+a \theta \tau}{1+\theta \tau}+2 \theta \frac{\tau(a-1)}{(1+\theta \tau)^{2}}\right] \frac{\mu_{S} b^{2}}{12}>0 \\
\Leftrightarrow(\theta \tau)^{2} a+(\theta \tau)(3 a-1)+1>0, \quad \forall(\theta \tau) .
\end{gathered}
$$

The last above inequality is verified only if we impose the condition

$$
\begin{gathered}
\Delta_{1}=(3 a-1)^{2}-4 a<0 \Leftrightarrow 9 a^{2}-10 a+1<0 \\
\Leftrightarrow a \in(1 / 9,1) .
\end{gathered}
$$

Therefore we get the following result:

$$
\mu+2 \theta \mu_{\theta}>0 \Leftrightarrow a \in(1 / 9,1) .
$$

The inequality $\mu+2 \theta \mu_{\theta}>0$ is quite similar with the restriction (4) of [8]. In this paper we consider the viscosity profiles which verify the condition (17).

We introduce the notation

$$
\gamma=k\left(1+2 \theta \mu_{\theta} / \mu\right)^{1 / 2},
$$

thus from (17) it follows $\gamma>0$. The relation (14) becomes

$$
-f_{x x}+\gamma^{2} f=0, \quad x \neq\{-L, 0\} .
$$

We need far decay perturbations, thus by using again the condition (17) (that means $\gamma>0$ ) we obtain

$$
f(x)=f(-L) \exp [(x+L) \gamma], x<-L,
$$




$$
\begin{gathered}
f(x)=f(0) \exp (-\gamma x), x>0, \\
f_{x}^{-}(-L)=\gamma f(-L), \quad f_{x}^{+}(0)=-\gamma f(0),
\end{gathered}
$$

where the indices,-+ stands for "left" and "right" limits.

The solution of (14) inside the intermediate region is

$$
f(x)=A e^{\gamma x}
$$

because a term of the form $B e^{-\gamma x}$ becomes very large for large positive $\gamma$ (recall $x<0$ ) and the amplitudes $f$ must be small in the frame of the linear stability analysis.

We study now the perturbed interfaces in $x=0, x=-L$. Near the point $x=0$ we consider the perturbed interface denoted by $g(x, y, t)$. As the interface is a material one, in the first approximation we get

$$
g_{t}=u^{\prime} \Rightarrow g(0, y, t)=[f(0) / \sigma] \exp (i k y+\sigma t)
$$

The perturbed pressure is obtained by using the Darcy law (1), the relation (13) and the first-order Taylor expansion for the basic pressure $P$ near $x=0$ :

$$
\begin{aligned}
& p^{+}(0)=P(0, y, t)+P_{x}^{+}(0, y, t) \cdot g(0, y, t)+p^{\prime}(0, y, t) \\
& \quad=P(0)-\mu^{+}(0)\left[\frac{U f(0)}{\sigma}+\frac{f_{x}^{+}(0)}{k^{2}}\right] \exp (i k y+\sigma t) .
\end{aligned}
$$

The same procedure is used to get the left limit of the pressure in $x=0$ and it follows

$$
p^{-}(0)=P(0)-\mu^{-}(0)\left[\frac{U f(0)}{\sigma}+\frac{f_{x}^{-}(0)}{k^{2}}\right] \exp (i k y+\sigma t) .
$$

A similar relation can be obtained for the point $x=-L$, which will be used in the relation (27) below.

On the interfaces $x=0, x=-L$ we consider the surface tensions $T(0), T(-L)$. We use the Laplace law and get

$$
\begin{aligned}
p^{+}(0)-p^{-}(0) & =T(0) g_{y y}(0, y, t), \\
p^{+}(-L)-p^{-}(-L) & =T(-L) g_{y y}(-L, y, t) .
\end{aligned}
$$

For simplicity, we will use notations

$$
\begin{gathered}
f_{0}=f(0), \quad f_{L}=f(-L), \quad T_{0}=T(0), \quad T_{L}=T(-L), \\
f_{x o}^{+,-}=f_{x}^{+,-}(0), \quad f_{x L}^{+,-}=f_{x}^{+,-}(-L),
\end{gathered}
$$




$$
\mu_{0}^{+,-}=\mu^{+,-}(0), \quad \mu_{L}^{+,-}=\mu^{+,-}(-L) .
$$

From relations $(22)-(24)$ it follows

$$
\begin{gathered}
\mu_{0}^{-}\left[\frac{U f_{0}}{\sigma}+\frac{f_{x 0}^{-}}{k^{2}}\right]-\mu_{0}^{+}\left[\frac{U f_{0}}{\sigma}+\frac{f_{x 0}^{+}}{k^{2}}\right]=-T_{0} \frac{f_{0}}{\sigma} k^{2}, \\
\mu_{L}^{-}\left[\frac{U f_{L}}{\sigma}+\frac{f_{x L}^{-}}{k^{2}}\right]-\mu_{L}^{+}\left[\frac{U f_{L}}{\sigma}+\frac{f_{x L}^{-}}{k^{2}}\right]=-T_{L} \frac{f_{L}}{\sigma} k^{2} .
\end{gathered}
$$

By direct calculations, as in [14], we obtain

$$
\begin{gathered}
\mu_{0}^{-} f_{x}^{-}(0)-\mu_{0}^{+} f_{x}^{+}(0)=\frac{k^{2} U\left[\mu_{0}^{+}-\mu_{0}^{-}\right]-k^{4} T_{0}}{\sigma} f_{0}, \\
\mu_{L}^{-} f_{x}^{-}(-L)-\mu_{L}^{+} f_{x}^{+}(-L)=\frac{k^{2} U\left[\mu_{L}^{+}-\mu_{L}^{-}\right]-k^{4} T_{L}}{\sigma} f_{L} .
\end{gathered}
$$

Therefore the interfaces stability is governed by the equation (14) and the boundary conditions (28), (29). We recall that $\mu$ is not depending on $x$; we consider

$$
\mu_{W}<\mu^{-}(0)=\mu^{+}(-L)=\mu(U)<\mu_{O} .
$$

All growth rates $\sigma$ must verify the equations (28) - (29).

We use the far filed conditions (19) for $f$. Thus all limit values of $f_{x}$ in $x=-L, x=0$ contains the factor $\gamma$ given by (18).

We compute $\sigma$ from both equations (28) - (29), we simplify with $\gamma$ in the denominators, and obtain

$$
\frac{k U\left(\mu-\mu_{W}\right)-k^{3} T_{0}}{\mu_{W}-\mu}=\frac{k U\left(\mu_{O}-\mu\right)-k^{3} T_{L}}{\mu+\mu_{O}} .
$$

We equate the coefficient of $k, k^{3}$, thus it follows

$$
\mu_{O}=0, \quad \mu=\mu_{W} T_{L} /\left(T_{L}+T_{0}\right) .
$$

Both above relations are in contradiction with our hypothesis:

i) The oil viscosity must be strictly positive.

ii) $\mu$ is depending on $U$ and is not depending on $T_{0}, T_{L}$.

As a consequence, the growth rates $\sigma$ can not exists. The stability problem (14), (28), (29) does not make sense. 


\section{When $\mu$ is depending on $U$ and $c$}

Let us consider a polymer-solute in the middle region, with a variable viscosity $\mu$ of the type

$$
\mu_{W}<\mu=\mu\left(c, U^{2}\right)<\mu_{O},
$$

where $c=c(x)$ is the polymer concentration. In [13] was pointed out that, for diluted polymer-solutes, $\mu$ is invertible with respect to $c$. Thus the viscosity $\mu$ in the middle region is depending also on $x$.

The perturbed viscosity is obtained as follows. We have

$$
\begin{aligned}
& \mu\left(c+c^{\prime},\left(U+u^{\prime}\right)^{2}+v^{\prime 2}\right) \\
\approx & \mu\left(c, U^{2}\right)+\mu_{c} c^{\prime}+\mu_{\theta} 2 U u^{\prime},
\end{aligned}
$$

therefore

$$
\mu^{\prime}=\mu_{c} c^{\prime}+\mu_{\theta} 2 U u^{\prime},
$$

where $c$ is the basic concentration profile and $c^{\prime}$ is the perturbed concentration.

As in [6], [7], we consider the following equation for $c$ in the fix coordinate system $x_{1} O y$

$$
c_{t}+U c_{x_{1}}=0 .
$$

We use the moving reference $x=x_{1}-U t$ and in the first approximation we get

$$
c_{t}^{\prime}=-c_{x} u^{\prime}, \quad c^{\prime}=-\frac{1}{\sigma} u^{\prime} c_{x},
$$

(see [6], relations (2.8) and (2.12) with $c$ instead of $\mu$ ). The equations (33) and (35) give us

$$
\begin{aligned}
\mu^{\prime} & =\left\{-\mu_{c} c_{x} / \sigma++\mu_{\theta} 2 U\right\} u^{\prime} \\
& =\left\{-\mu_{x} / \sigma++\mu_{\theta} 2 U\right\} u^{\prime} .
\end{aligned}
$$

The equations (9)-(10) still hold. We use the expression (36) and obtain

$$
\begin{gathered}
p_{x}^{\prime}=-\mu u^{\prime}-U \mu^{\prime} \\
=-\mu u^{\prime}-U\left(-\mu_{x} / \sigma+2 \mu_{\theta} U\right) u^{\prime}, \\
p_{y}^{\prime}=-\mu v^{\prime} .
\end{gathered}
$$

The relation $\left(p_{x}^{\prime}\right)_{y}=\left(p_{y}^{\prime}\right)_{x}$ gives us the amplitude equation inside the middle region $(-L, 0)$ :

$$
k^{2}\left(-\mu+U \mu_{x} / \sigma-2 \theta \mu_{\theta}\right) f=-\left(\mu f_{x}\right)_{x},
$$




$$
-\left(\mu f_{x}\right)_{x}+k^{2}\left(\mu+2 \theta \mu_{\theta}\right) f=k^{2} U \mu_{x} f / \sigma .
$$

This time we do not know the exact expression of $f$ inside the middle region, but we have the same relations (19) for $f$ in the far field (where $\mu_{x}=0$ ). The boundary conditions (26), (27) still hold.

We multiply the relation (39) with $f$, we integrate on $(-L, 0)$ and obtain

$$
\begin{gathered}
-\left(\mu^{-} f_{x}^{-} f\right)(0)+\left(\mu^{+} f_{x}^{+} f\right)(-L)+\int \mu f_{x}^{2} d x \\
+k^{2} \int\left(\mu+2 \theta \mu_{\theta}\right) d x=k^{2} \frac{U}{\sigma} \int \mu_{x} f^{2} d x
\end{gathered}
$$

where we used the notations

$$
(F G H)(x)=F(x) H(x) F(x), \quad \int F d x=\int_{-L}^{0} F(x) d x .
$$

The boundary conditions (28), (29) and (40) give us:

$$
\begin{gathered}
-\left[k E_{0} f_{0}^{2} / \sigma-\gamma \mu_{0}^{+} f_{0}^{2}\right]+\left[\gamma \mu_{L}^{-} f_{L}^{2}-k E_{L} f_{L}^{2} / \sigma\right] \\
+\int \mu f_{x}^{2} d x+k^{2} \int\left(\mu+2 \theta \mu_{\theta}\right) d x=k^{2} \frac{U}{\sigma} \int \mu_{x} f^{2} d x, \\
\mu_{0}^{+,-}=\mu^{+,-}(0), \quad \mu_{L}^{-,+}=\mu^{-,+}(-L), \\
E_{0}=k U\left[\mu_{O}-\mu_{0}^{-}\right] k^{3} T_{0}, \\
E_{L}=k U\left[\mu_{L}^{+}-\mu_{W}\right]-k^{3} T_{L} .
\end{gathered}
$$

From the above relations (41) we obtain

$$
\sigma=\frac{k E_{0} f_{0}^{2}+k E_{L} f_{L}^{2}+k^{2} U \int \mu_{x} f^{2} d x}{\mu_{0} \gamma f_{0}^{2}+\mu_{W} \gamma f_{L}^{2}+\int \mu f_{x}^{2} d x+k^{2} \int\left(\mu+2 \theta \mu_{\theta}\right) f^{2} d x} .
$$

We consider now a continuous viscosity and zero surfaces tensions, that means

$$
E_{0}=E_{L}=T_{0}=T_{L}=0 .
$$

For a Newtonian intermediate liquid depending only on $c$, the following estimate of the growth rates (say, $\sigma_{c}$ ) is given in formula (44) of [13]:

$$
\sigma_{c} \leq \frac{U \int \mu_{x} f^{2} d x}{\int \mu f^{2} d x}
$$


From (42) we get the upper estimate

$$
\sigma \leq \frac{U \int \mu_{x} f^{2} d x}{\int\left(\mu+2 \theta \mu_{\theta}\right) f^{2} d x}
$$

The upper limit (45) is less than the upper bound (44) when $\mu_{\theta}>0$. Therefore we get an improved stability in the non-Newtonian case, if the hypothesis (43) is fulfilled.

Remark. For small enough $\mu_{x}$ and large enough $\mu_{\theta}$ (recall $\theta=U^{2}$, the upper bound (45) becomes arbitrary small. Thus we can almost suppress the Saffman-Taylor instability, even if the surface tensions are zero. We recall the Saffman-Taylor growth rate $\sigma_{S T}$ for water displacing oil with a surface tension $T$ :

$$
\sigma_{S T}=\frac{k U\left(\mu_{O}-\mu_{W}\right)-k^{3} T}{\mu_{O}+\mu_{W}} .
$$

We have

$$
T=0 \Rightarrow \lim _{k \rightarrow \infty} \sigma_{S T}=\infty
$$

\section{References}

[1] D. Bonn, H. Kellay, M. Brunlich, M. Amar, J. Meunier, Viscous fingering in complex fluids, Physica A: Statistical Mechanics and its Applications, 220, No 1-2 (1995), 60-73; doi: 10.1016/0378-4371(95)00114-M.

[2] D. Bonn, H. Kellay, M. Amar, J. Meunier, Viscous finger widening with surfactants and polymers. Physical Review Letters, 75, No 11 (1995), 21322135; doi: 10.1103/PhysRevLett.75.2132.

[3] C. Carasso and G. Paşa, An optimal viscosity profile in the secondary oil recovery, Mod. Math. et Analyse Numérique, 32, No 2 (1998), 211-221; $\mathrm{id}=\mathrm{M} 2 \mathrm{AN}-1998-32-2-211-0$.

[4] P. Daripa and G. Pasa, Stabilizing effect of diffusion in enhanced oil recovery and three-layer Hele-Shaw flows with viscosity gradient, Transp. in Porous Media, 70, No 1 (2007), 11-23; doi: 10.1007/s11242-007-9122-7.

[5] P. Daripa and G. Pasa, On diffusive slowdown in three-layers Hele-Shaw flows, Quarterly of Appl. Math., LXVIII, No 33 (2010), 591-606; doi: 10.1090/S0033-569X-2010-01174-3. 
[6] S.B. Gorell and G.M. Homsy, A theory of the optimal policy of oil recovery by secondary displacement process, SIAM J. Appl. Math., 43, No 1 (1983), 79-98; doi: 10.1137/0143007.

[7] S.B. Gorell and G.M. Homsy, A theory for the most stable variable viscosity profile in graded mobility displacement process, AIChE Journal, 31, No 9 (1985), 1598-1503; doi: 10.1002/aic.690310912.

[8] L. Kondic, P. Palffy-Muhoray, and M.J. Shelley, Models of nonNewtonian Hele-Shaw flow, Phys. Rev. E, 54 No 5 (1996), 4536-4539; doi: 10.1103 / physreve.54r4536.

[9] R. Niu, H. Zheng, B. Zhang, Navier-Stokes equations the half-space in variable exponent spaces of Clifford-valued functions, Electron. J. Differential Equations, 2017, No 98 (2017), 1-21; doi: 10.1186/s13661-015-0291-y.

[10] G. Pasa and O. Titaud, A class of viscosity profiles for oil displacement in porous media or Hele-Shaw cell, with Olivier Titaud, Transp. in Porous Media, 58, No 3 (2005), 269-286; doi: 10.1007/s11242-004-0773-3.

[11] G. Pasa, Stability analysis of diffusive displacement in three-layer HeleShaw cell or porous medium, Transport in Porous Media, 85, No 1 (2010), 317-332; doi: 10.1007/s11242-010-9564-1.

[12] G. Pasa, n the displacement of two immiscible Oldroyd-B fluids in a Hele-Shaw cell, Ann. Univ. Ferrara, 65, No 2 (2019), 337-359; doi: 10.1007/s11565-019-00320-7.

[13] G. Pasa, A paradox in Hele-Shaw displacements, Ann. Univ. Ferrara, 66, No 1 (2020), 99-111; doi: 10.1007/s11565-020-00339-1.

[14] G. Pasa, The multi-layer Hele-Shaw model with constant viscosity fluids can not minimize the Saffman-Taylor instability, Internaional Journal of Applied Mathematics, 33, No 4 (2020), 697-708; doi: 10.12732/ijam.v33i4.13.

[15] P.G. Saffman and G.I. Taylor, The penetration of a fluid in a porous medium or Helle-Shaw cell containing a more viscous fluid, Proc. Roy. Soc. A, 245, No 1242 (1958), 312-329; doi: 10.1098/rspa.1958.0085.

[16] S.D.R. Wilson, The Taylor-Saffman problem for a non-Newtonian liquid, J. Fluid Mech. 220 (1990), 413-425; doi: 10.1017/S0022112090003329. 
